\title{
Robust Multi-Objective PQ Scheduling for Electric Vehicles in Flexible Unbalanced Distribution Grids
}

\author{
Knezovic, Katarina; Soroudi, Alireza; Marinelli, Mattia; Keane, Andrew
}

Published in:

IET Generation Transmission and Distribution

Link to article, DOI:

10.1049/iet-gtd.2017.0309

Publication date:

2017

Document Version

Peer reviewed version

Link back to DTU Orbit

Citation (APA):

Knezovic, K., Soroudi, A., Marinelli, M., \& Keane, A. (2017). Robust Multi-Objective PQ Scheduling for Electric Vehicles in Flexible Unbalanced Distribution Grids. IET Generation Transmission and Distribution, 11(16), 40314040. https://doi.org/10.1049/iet-gtd.2017.0309

\section{General rights}

Copyright and moral rights for the publications made accessible in the public portal are retained by the authors and/or other copyright owners and it is a condition of accessing publications that users recognise and abide by the legal requirements associated with these rights.

- Users may download and print one copy of any publication from the public portal for the purpose of private study or research.

- You may not further distribute the material or use it for any profit-making activity or commercial gain

- You may freely distribute the URL identifying the publication in the public portal 


\title{
Robust Multi-Objective PQ Scheduling for Electric Vehicles in Flexible Unbalanced Distribution Grids
}

\author{
Katarina Knezović $^{1, *}$, Alireza Soroudi ${ }^{2}$, Andrew Keane $^{2}$, Mattia Marinelli $^{1}$ \\ ${ }^{1}$ Centre for Electric Power and Energy, Department of Electrical Engineering, Technical University \\ of Denmark, Denmark \\ ${ }^{2}$ School of Electrical and Electronic Engineering, University College Dublin, Dublin, Ireland \\ "kknez@elektro.dtu.dk
}

\begin{abstract}
With increased penetration of distributed energy resources and electric vehicles (EVs), different EV management strategies can be used for mitigating adverse effects and supporting the distribution grid. This paper proposes a robust multi-objective methodology for determining the optimal day-ahead EV charging schedule while complying with unbalanced distribution grid constraints. The proposed methodology considers partially competing objectives of an EV aggregator and the respective distribution system operator, and applies a fuzzy-based mechanism for obtaining the best compromise solution. The robust formulation effectively considers the errors in the electricity price forecast and its influence on the EV schedule. Moreover, the impact of EV reactive power support on objective values and technical parameters is analysed both when EVs are the only flexible resources and when linked with other demand response programs. The method is tested on a real Danish unbalanced distribution grid with 35\% EV penetration to demonstrate the effectiveness of the proposed approach. It is shown that the proposed formulation guarantees an optimal EV cost as long as the price uncertainties are lower than the aggregator's conservativeness degree, and that EV reactive power improves local conditions without significantly affecting the EV cost.
\end{abstract}




\section{Nomenclature}

\section{Sets and Indices}

$\phi \quad$ Set of phases $\{a, b, c\}$.

$i, j \quad$ Index for bus $\mathrm{i}, \mathrm{j}$.

$l \quad$ Set of distribution lines.

$t \quad$ Set of time intervals.

\section{Parameters}

$(P / Q)_{0, i}^{\phi D}$ Nominal active/reactive power of demand connected to bus $i$ on phase $\phi$.

$\Delta_{t}^{-/+}$Negative/positive electricity price deviation at time $t$.

$\eta_{c h, i}^{\phi, E V}$ Charging efficiency of an EV connected to bus $i$ on phase $\phi$.

$\Gamma \quad$ Conservativeness degree of the decision maker with respect to price uncertainty.

$\lambda_{t}^{f / a} \quad$ Forecasted/actual electricity price at time $t$.

$\lambda_{t}^{\min / \max }$ Minimum/maximum electricity price bound at time $t$.

$\left|V_{i, t, \min / \max }^{\phi}\right|$ Minimum/maximum acceptable voltage magnitude of phase $\phi$ at bus $i$ at time t.

$\left|Y_{i j}^{\phi_{1} \phi_{2}-n}\right|$ Admittance magnitude between phase $\phi_{1}$ at bus $i$ and phase $\phi_{2}$ at bus $j$ of branch $i j$.

$\overline{\lambda_{t}} \quad$ Uncertain electricity price at time $t$.

$\theta_{i j}^{\phi_{1} \phi_{2}} \quad$ Admittance angle between phase $\phi_{1}$ at bus $i$ and phase $\phi_{2}$ at bus $j$ of branch $i j$.

$\varphi_{i, t}^{\phi D} \quad$ Power factor of demand connected to bus $i$ on phase $\phi$ at time t.

$\xi_{i} \quad$ Demand flexibility parameter for bus $\mathrm{i}$.

$k_{i}^{\phi E V}$ Converter parameter for reactive power control of an EV connected to bus $i$ on phase $\phi$.

$P_{i, \max }^{\phi}$ Maximum active power of an EV connected to bus $i$ on phase $\phi$. 
$Q_{i, \min / \max }^{\phi}$ Minimum/maximum reactive power of an EV connected to bus $i$ on phase $\phi$.

$S_{i j, \max }^{\phi}$ Maximum apparent power on phase $\phi$ of branch $i j$.

$S O C_{0 / \max , i, t}^{\phi E V}$ Initial/maximum state of charge of an EV connected to bus $i$ on phase $\phi$ at time $t$.

$t_{\text {start/end,i }}^{\phi E V}$ Arrival/departure time of an EV connected to bus $i$ on phase $\phi$ at time $t$.

\section{Variables}

$(P / Q)_{i, t}^{\phi \text { Dnew }}$ Active/reactive power of demand connected to bus $i$ on phase $\phi$ at time t.

$(P / Q)_{i, t}^{\phi E V}$ Active/reactive power of an EV connected to bus $i$ on phase $\phi$ at time $\mathrm{t}$.

$(P / Q)_{i, t}^{\phi G}$ Active/reactive power of a generating unit connected to bus $i$ on phase $\phi$ at time t.

$\beta, \omega_{t}, \Theta$ Auxiliary variables.

$\delta_{i}^{\phi} \quad$ Voltage angle of phase $\phi$ at bus $i$.

$\left|V_{i, t}^{\phi}\right|$ Voltage magnitude of phase $\phi$ at bus $i$ at time $\mathrm{t}$.

$P_{0, i, t}^{\phi E V}$ Active power of an EV connected to bus $i$ on phase $\phi$ at time $\mathrm{t}$ at nominal voltage conditions.

$S O C_{i, t}^{\phi E V}$ State of charge of an EV connected to bus $i$ on phase $\phi$ at time $t$.

\section{Introduction}

Fundamental changes occurring in the electric power system promoted by the global sustainability efforts are reshaping the electrical grid operation. With increased penetration of distributed energy resources, there is an additional need for control strategies which allow them to provide various flexibility services and avoid over-investments for maintaining the grid security [1]. Additionally, since the market share of electric vehicles (EVs) is expected to grow significantly in the following years, even greater system complexity is imposed [2]. Uncontrolled EV charging may result in voltage violations and cable overloading followed by the need for grid reinforcement, but also in increased operational cost due to increased energy losses. As an economic alternative, different EV charging strategies can be used for mitigating the adverse effects and supporting the grid. 
An extensive amount of research has been made on coordinated EV charging proving that such concept can be used for lowering the undesired impacts on the power system and providing ancillary services [3-5]. A new business entity, namely EV aggregator, has been widely proposed to coordinate large EV amounts and offer their services to system operators via centralised control which is proven to reduce losses, improve voltage stability and decrease peak loading compared to the uncontrolled case. Various studies use optimal power flow formulation for EV scheduling in order to minimise the charging cost or maximise the EV aggregator revenue [6,7], but they usually deal with large EV numbers at the transmission level and completely omit distribution grid constraints. In a smart grid context, obtaining an optimal EV charging schedule requires an adequate grid representation since results must be feasible in the respective grid with corresponding constraints [8-10]. In addition to EV active power scheduling, EV reactive power can also be used to support the distribution grid. Compared to traditional voltage regulation means in distribution grids such as capacitor banks and on-load tap changers [11], EV reactive power capability can provide dynamic, continuous and distributed support as well as inductive reactive power support if and where needed. For instance, autonomous reactive power support based on droop control has been investigated in $[12,13]$, but the approaches do not provide any optimal scheduling. Moreover, even though they are cost-effective, active and reactive power droop controllers may cause undesirable avalanche effects of simultaneous reactions [14]. So, more advanced methods with direct communication to service providers are needed to unleash the full demand response potential, including EVs [15]. Ref. [16] presents a PQ optimisation method for EV (dis)charging, but focuses only on the total charging cost, whereas distribution grid constraints have been completely disregarded. These constraints have been taken into account in $[17,18]$, but only for balanced systems. Similarly, [19,20] deal with robust EV scheduling, but they either disregard the distribution grid constraints or only consider balanced conditions.

As pointed out in [3], combining several objective functions in EV scheduling has scarcely been touched upon, even though combining both DSO's and EV aggregator's concern is of utter importance. In [21], the proposed multi-objective framework combines minimisation of EV charging cost and minimisation of reactive power insufficiency, but specific power system results 
are not presented, and it remains unclear if the framework is applicable to unbalanced systems. Ref. [22] proposes a multi-objective formulation at the distribution level for minimising operation cost and voltage deviations, but it is applicable only to balanced grids. Since distribution systems are usually unbalanced and EVs are single-phase connected, the individual EV charging schedules do not have to coincide for all vehicles, especially in heavily unbalanced networks. Hence, using unbalanced optimal power flow is essential in order to accurately represent the distribution system and the unbalance effect on the operation schedules. In [23], unbalanced constraints are taken into account, but the impact of electricity price uncertainty is disregarded.

Even though EV smart charging problem is well studied and numerous approaches are proposed for achieving this behaviour, many existing methods suffer from one or more of the following drawbacks: (1) lack of distribution grid constraints, (2) optimal power flow formulation for balanced grids, (3) no EV reactive power flexibility, (4) no multi-objective formulation for collaborative EV scheduling between the DSO and the EV aggregator, and (5) disregarding the uncertainties associated to the electricity price. To the authors' knowledge, the existing research has not looked into combining all of the mentioned aspects together. The contributions of this paper are as follows:

- To propose a novel model for obtaining a combined EV active and reactive power day-ahead schedule considering unbalanced distribution grid constraints. Using the unbalanced optimal power flow allows to schedule the individual EVs with respect to the constraints of the phase they are connected to.

- To propose a novel model with a multi-objective formulation which combines two partially competing objectives: minimisation of the DSO's losses which represent the local grid efficiency, and minimisation of EV aggregator's charging cost which represent the system-wide aspect as the EV aggregator participates in the wholesale electricity market. The methodology provides not only one solution, but a set of solutions from which an optimal schedule can be chosen with a proper balance between the DSO's and EV aggregator's concerns.

- To consider the uncertainty in electricity price which influences EV charging costs. By using the robust optimisation technique, it is guaranteed that EV schedules remain acceptable if the 
actual electricity price deviates from the forecasted value to a certain conservativeness degree defined by the EV aggregator.

- To analyse the impact of EV reactive power support both on technical parameters and on EV charging cost in case when EVs are the only flexible resource and when interconnected with other demand response.

\section{Problem formulation and modelling}

\subsection{Assumptions}

The assumptions of this paper are described as follows:

- All EVs are under the jurisdiction of a single EV aggregator who entered into a contract with individual $\mathrm{EV}$ owners, knows their connection points and uses estimation techniques for predicting EV arrival and departure times to manage the scheduling. EVs are equipped with smart metering technology and can be remotely controlled by receiving the active/reactive power charging set point. It is also assumed that EV users are not interested in how and when the vehicles are charged as long as they are fully available by the estimated departure time which remains true as long as the aggregator remunerates all users in the same manner. The specific way the aggregator chooses to remunerate the users is beyond the scope of this paper.

- Grid operator has access to the following information: network size, network topology, line specifications and transformer specifications. Smart metering technology with load control capability is assumed to be present in each household and can be used for rescheduling part of the consumption through demand response program [1,24].

- DSO and EV aggregator utilise techniques for forecasting the day-ahead electricity price with the respective uncertainty bounds, as well as the consumption which can be forecasted with reasonable accuracy. Therefore, the error associated with the load forecast and user behaviour has been disregarded.

- Similarly to available PV inverters, the 4-quadrant EV converter can be enabled to exchange 
reactive power with the grid without affecting the state-of-charge and consequently user comfort. It is assumed that $\mathrm{EV}$ converters are sized to provide reactive power additionally to the active power charging rate with no need for curtailing the active power $[12,13]$. Similar PV inverters are already commercially available due to grid codes in several European countries.

\subsection{Constraints}

In this work, a three-phase grounded four-wire system optimal power flow is formulated based on [25] and implemented as a single non-linear program which can be solved by commercial non-linear solvers such as CONOPT or IPOPT. Within this formulation, the calculated active and reactive power for phase $a$ of branch $i j$ at time $t$ are given as follows:

$$
\begin{aligned}
& P_{i j, t}^{a}=\sum_{\phi=a, b, c}\left(\left|V_{i, t}^{a}\right|\left|Y_{i j}^{a \phi-n}\right|\left|V_{i, t}^{\phi}\right| \cos \left(\theta_{i j}^{a \phi}+\delta_{i, t}^{\phi}-\delta_{i, t}^{a}\right)-\left|V_{i, t}^{a}\right|\left|Y_{i j}^{a \phi-n}\right|\left|V_{j, t}^{\phi}\right| \cos \left(\theta_{i j}^{a \phi}+\delta_{j, t}^{\phi}-\delta_{i, t}^{a}\right)\right) \\
& Q_{i j, t}^{a}=\sum_{\phi=a, b, c}\left(\left|V_{i, t}^{a}\right|\left|Y_{i j}^{a \phi-n}\right|\left|V_{j, t}^{\phi}\right| \sin \left(\theta_{i j}^{a \phi}+\delta_{j, t}^{\phi}-\delta_{i, t}^{a}\right)-\left|V_{i, t}^{a}\right|\left|Y_{i j}^{a \phi-n}\right|\left|V_{i, t}^{\phi}\right| \sin \left(\theta_{i j}^{a \phi}+\delta_{i, t}^{\phi}-\delta_{i, t}^{a}\right)\right)
\end{aligned}
$$

Similar equations can be extracted for active and reactive power of the remaining two phases $b$ and $c$. The power mismatch equations for each bus are given as follows:

$$
\begin{aligned}
& \sum_{\substack{j=1 \\
j \neq i}}^{N_{j}} P_{i j, t}^{\phi}=\sum_{G=1}^{N_{G}} P_{i, t}^{\phi G}-\sum_{D=1}^{N_{D}} P_{i, t}^{\phi \text { Dnew }}-\sum_{E V=1}^{N_{E V}} P_{i, t}^{\phi E V} \\
& \sum_{\substack{j=1 \\
j \neq i}}^{N_{j}} Q_{i j, t}^{\phi}=\sum_{G=1}^{N_{G}} Q_{i, t}^{\phi G}-\sum_{D=1}^{N_{D}} Q_{i, t}^{\phi \text { Dnew }}-\sum_{E V=1}^{N_{E V}} Q_{i, t}^{\phi E V}
\end{aligned}
$$

The voltage dependency of residential demand is given by equation (5) where $P_{0, i}^{\phi D}$ and $Q_{0, i}^{\phi D}$ represent the load's nominal active and reactive power, respectively, whereas $\kappa$ equals to zero for constant power loads, one for constant current loads or two for constant impedance loads. Furthermore, residential consumption is assumed to be somewhat flexible, so the load may vary within the 
observed period as described by equation (6) and equation (7). The load's reactive power is then given by equation (8).

$$
\begin{aligned}
& P_{i, t}^{\phi D}=P_{0, i}^{\phi D} \cdot\left|V_{i, t}^{\phi}\right|^{\kappa} \\
& \sum_{t} P_{i, t}^{\phi \text { Dnew }} \cdot\left|V_{i, t}^{\phi}\right|^{\kappa}=\sum_{t} P_{0, i}^{\phi D} \cdot\left|V_{i, t}^{\phi}\right|^{\kappa} \\
& \left(1-\xi_{i}\right) P_{0, i}^{\phi D} \leq P_{i, t}^{\phi \text { Dnew }} \leq\left(1+\xi_{i}\right) P_{0, i}^{\phi D} \\
& Q_{i, t}^{\phi \text { Dnew }}=\tan \left(\arccos \left(\varphi_{i, t}^{\phi D}\right)\right) \cdot P_{i, t}^{\phi \text { Dnew }} \cdot\left|V_{i, t}^{\phi}\right|^{\kappa}
\end{aligned}
$$

The distribution grid voltage and power flow constraints are formulated as follows:

$$
\begin{aligned}
& V_{i, t, \min }^{\phi} \leq\left|V_{i, t}^{\phi}\right| \leq V_{i, t, \max }^{\phi} \\
& \left(P_{i j, t}^{\phi}\right)^{2}+\left(Q_{i j, t}^{\phi}\right)^{2} \leq\left(S_{i j, \max }^{\phi}\right)^{2}
\end{aligned}
$$

where $S_{i j, \max }^{\phi}$ is the maximum apparent power capacity of branch $i j$. In addition, the MV side of the transformer is assumed to be the slack bus with fixed voltage magnitudes and angles.

EV characteristics are expressed using the following constraints:

$$
\begin{aligned}
& S O C_{i, t}^{\phi E V}=S O C_{i, t-1}^{\phi E V}+P_{i, t}^{\phi E V} \cdot \Delta t \cdot \eta_{c h, i}^{\phi E V} \\
& S O C_{0, i}^{\phi E V} \leq S O C_{i, t}^{\phi E V} \leq S O C_{i, \max }^{\phi, E V} \\
& S O C_{i, t \mid t=t_{\text {end }}}^{\phi E V}=S O C_{i, \max }^{\phi, E V} \\
& P_{i, t}^{\phi E V}=P_{0, i, t}^{\phi E V} \cdot\left|V_{i, t}^{\phi}\right| \\
& 0 \leq P_{0, i, t}^{\phi E V} \leq P_{i, \max }^{\phi, E V} \\
& -k_{i, t}^{\phi E V} \cdot P_{i, t}^{\phi E V} \leq Q_{i, t}^{\phi E V} \leq k_{i, t}^{\phi E V} \cdot P_{i, t}^{\phi E V} \\
& Q_{i, \min }^{\phi, E V} \leq Q_{i, t}^{\phi E V} \leq Q_{i, \max }^{\phi, E V}
\end{aligned}
$$

Equation (11) describes EV state of charge (SOC) dependent on the SOC in the previous time step, EV charging power and EV charging efficiency, whereas the battery size constraint is given 
by equation (12). Equation (13) imposes the restriction where EVs must be fully charged before the estimated departure time to ensure they are fully available for primary transportation purposes. As represented in equation (14), EVs are modelled as a constant current load with $\kappa=1[26,27]$ where $P_{0, i, t}^{\phi E V}$ represents the $\mathrm{EV}$ active power value at nominal voltage conditions. In addition to EV active power constraints described in equation (15), it is assumed that EVs have the possibility to dynamically modulate the power factor under constraints described in equation (16) and equation (17). $k_{i, t}^{\phi E V}$ is fixed for each $\mathrm{EV}$ converter, e.g., $k_{i, t}^{\phi E V}=1 / 3$ for a converter capable of modulating the power factor up to 0.95 (ind./cap.).

\subsection{Nominal optimisation problem}

The proposed methodology obtains an optimal EV active and reactive schedule considering two partially competing objective functions which combine both the DSO's and the EV aggregator's concerns in one multi-objective model. The first objective is minimising the operating cost in terms of energy losses [28] which represents one of the main DSO concerns. The minimisation of energy losses $f_{1}$ can be formulated as:

$$
\min f_{1}=\sum_{t} \sum_{l=1}^{N_{l}} \sum_{\phi=a, b, c} P_{l, t}^{\phi l o s s}=\sum_{t} \sum_{i}^{N_{i}} \sum_{j}^{N_{j}} \sum_{\phi=a, b, c}\left(P_{i j, t}^{\phi}+P_{j i, t}^{\phi}\right)
$$

where $P_{l, t}^{\phi l o s s}$ are the total losses on phase $\phi$ of line $l$.

The second objective function is minimising the total EV charging cost since it is assumed that the aggregator enters into a contract with individual EV owners. Then, this function represents the aggregator's main concern as by minimising the total charging cost, it maximises the revenue. The minimisation of $\mathrm{EV}$ charging cost $f_{2}$ can be formulated as:

$$
\min f_{2}=\sum_{t} \sum_{E V=1}^{N_{E V}} \sum_{\phi=a, b, c} P_{i, t}^{\phi E V} \lambda_{t}
$$

Assuming that $F(\mathbf{D V}, \Pi)$ is the vector of objective functions where $\mathbf{D V}$ represents decision variables and $\Pi$ represents input parameters, whereas $H(\mathbf{D V}, \Pi)$ and $G(\mathbf{D V}, \Pi)$ represent equality 
and inequality constraints, respectively, the proposed multi-objective minimisation problem can generally be formulated as follows:

$$
\begin{array}{ll}
\underset{\mathbf{D V}}{\operatorname{minimise}} & F(\mathbf{D V}, \Pi)=\left[f_{1}(\mathbf{D V}, \Pi), f_{2}(\mathbf{D V}, \Pi)\right] \\
\text { subject to: } & \{G(\mathbf{D V}, \Pi)=0, H(\mathbf{D V}, \Pi) \leq 0\}
\end{array}
$$

For solving the multi-objective problem and obtaining the Pareto optimal front, $\epsilon$-constraint method is used due to several advantages [29], e.g., it can be used for both convex and non-convex Pareto optimal sets, it does not require scaling of the objective functions which can influence the results, and it needs less iterations for the front discovery compared to the weighted-sum method where several weight combinations can result in the same solution. The $\epsilon$-constraint method involves minimising the primary objective function while expressing the other objective in a form of inequality constraint. Equation (20) can then be reformulated as follows:

$$
\begin{array}{cl}
\underset{\mathbf{D V}}{\operatorname{minimise}} & f_{2}(\mathbf{D V}, \Pi) \\
\text { subject to: } & \{G(\mathbf{D V}, \Pi)=0, H(\mathbf{D V}, \Pi) \leq 0\} \\
& f_{1}(\mathbf{D V}, \Pi) \leq \epsilon
\end{array}
$$

where $\epsilon$ varies from the maximum to the minimum value of $f_{1}$.

After obtaining all Pareto points and in case the front is non-convex, it is necessary to compare individual solutions and exclude the dominated ones (local Pareto points) from the frontier.

\subsection{Uncertainty modelling}

There are several techniques for modelling the price uncertainty including stochastic scenario modelling, fuzzy modelling, Info-gap decision theory and robust optimisation [30]. In this work, robust optimisation is used to handle the uncertainties which are defined as an interval around the forecasted value. The idea of robust optimisation is to minimise the objective function without knowing the exact electricity price value, but only its minimum and maximum bounds. Such intervals are usually obtained using time-series models (ARIMA), neural networks and historic data 
165

[31]. Hence, the uncertain electricity price is formulated as follows:

$$
\bar{\lambda}_{t} \in U\left(\bar{\lambda}_{t}\right)=\left\{\bar{\lambda}_{t}: \lambda_{t}^{\min } \leq \bar{\lambda}_{t} \leq \lambda_{t}^{\max }\right\}
$$

where $\lambda_{t}^{\min }$ and $\lambda_{t}^{\max }$ are the lower and upper bounds of $\bar{\lambda}_{t}$, respectively.

Optimal decision making is done so that the obtained solution remains good if the actual electricity price $\lambda_{t}^{a}$ deviates from the forecasted value $\lambda_{t}^{f}$ to some degree $\Gamma$. Ref. [32] proves that the robust solution will be feasible with high probability even when more than $\Gamma$ forecasting errors occur. In case the actual electricity price is higher than the forecasted value, constraints for uncertainty modelling can be expressed as equation (23). Similarly, in case the electricity price is lower than the forecasted value, constraints in equation (24) apply. As the decision maker seeks the robustness against undesired events, equation (24) is not considered as an issues, so the main concern remains the case when the electricity price is higher than the forecasted values, as formulated in equation (23).

$$
\begin{aligned}
& \lambda_{t}^{a}=\lambda_{t}^{f}+\Delta_{t}^{+} \omega_{t} \\
& \Delta_{t}^{+}=\lambda_{t}^{\max }-\lambda_{t}^{f} \\
& 0 \leq \omega_{t} \leq 1
\end{aligned}
$$

$$
0 \leq \omega_{t} \leq 1
$$




\subsection{Robust optimisation formulation}

Taking price uncertainty into consideration, the objective function formulated in (19) becomes:

$$
\begin{array}{ll}
\underset{\mathbf{D V}}{\operatorname{minimise}} & f_{2}\left(\mathbf{D V}, \Pi, \overline{\lambda_{t}}\right)=\sum_{t} \sum_{E V=1}^{N_{E V}} \sum_{\phi=a, b, c}\left(P_{i, t}^{\phi E V} \lambda_{t}^{f}+P_{i, t}^{\phi E V} \Delta_{t}^{+} \omega_{t}\right) \\
& 0 \leq \omega \leq 1 \\
& \sum_{t} \omega_{t} \leq \Gamma
\end{array}
$$

subject to:

$$
\begin{aligned}
& \text { (1) to }(17) \\
& f_{1}(\mathbf{D V}, \Pi) \leq \epsilon
\end{aligned}
$$

Here, $\Gamma$ is a parameter specified by the decision maker which is introduced to prevent too conservative solutions [32]. More precisely, it denotes the maximum total deviation that can be tolerated. $\Gamma$ can vary from 0 (meaning no uncertainty may happen) to 24 (all uncertain parameters may take their worst value). The higher the $\Gamma$, the more conservative the decision maker is. One should note how for $\Gamma=0$, the robust problem converts into the nominal one. In order to find the worst case condition of price uncertainty that would cause the maximum increase in EV cost, it is necessary to formulate the robust counter part of equation (25) as follows [33]:

$$
\underset{\mathbf{D V}}{\operatorname{minimise}} \quad f_{2}\left(\mathbf{D V}, \Pi, \overline{\lambda_{t}}\right)=\sum_{t} \sum_{E V=1}^{N_{E V}} \sum_{\phi=a, b, c}\left(P_{i, t}^{\phi E V} \lambda_{t}^{f}+\max _{\omega_{t}}\left\{\begin{array}{c}
P_{i, t}^{\phi E V} \Delta_{t}^{+} \omega_{t} \\
\text { s.t: }(25 \mathrm{~b}),(25 \mathrm{c})
\end{array}\right\}\right)
$$

subject to: (1) to (17)

$$
f_{1}(\mathbf{D V}, \Pi) \leq \epsilon
$$


This formulation requires to solve a bi-level optimisation problem which can, according to the duality gap theory [33], be transformed into:

$$
\begin{array}{ll}
\underset{\mathbf{D V}}{\operatorname{minimise}} & f_{2}\left(\mathbf{D V}, \Pi, \overline{\lambda_{t}}\right)=\sum_{t} \sum_{E V=1}^{N_{E V}} \sum_{\phi=a, b, c} P_{i, t}^{\phi E V} \lambda_{t}^{f}+\sum_{t} \beta_{t}+\Gamma \Theta \\
& \Theta+\beta_{t} \geq\left(\lambda_{t}^{\max }-\lambda_{t}^{f}\right) \cdot \sum_{E V=1}^{N_{E V}} \sum_{\phi=a, b, c} P_{i, t}^{\phi E V} \\
& \Theta, \beta_{t} \geq 0
\end{array}
$$

subject to:

(1) to $(17)$

$$
f_{1}(\mathbf{D V}, \Pi) \leq \epsilon
$$

We remark that the obtained single level optimisation in equation (27) can also be applied to other scenarios with different sources of uncertainty. For instance, price deviations can be formulated to represent the error associated to load uncertainty or EV availability [34].

\subsection{Best compromise solution}

Once the Pareto optimal front is determined, a range of solutions is available between which the final operating schedule should be chosen. Here, a fuzzy satisfying set theory is used to choose the best candidate solution. The concept can be described as follows: for each solution $X_{n}$ in the Pareto optimal front with $N_{s}$ solutions, a membership function $\mu_{k}\left(X_{n}\right)$ is defined to show the level of which $X_{n}$ belongs to the set that minimises the objective function $f_{k}$. A linear membership function is used for both objective functions as follows:

$$
\forall k \in\{1,2\} \mu_{k}\left(X_{n}\right)= \begin{cases}0, & f_{k}\left(X_{n}\right)>f_{k \max } \\ \frac{f_{k \max }-f_{k}\left(X_{n}\right)}{f_{k \max }-f_{k \min }}, & f_{k \min } \leq f_{k}\left(X_{n}\right) \leq f_{k \max } \\ 1, & f_{k}\left(X_{n}\right)<f_{k \min }\end{cases}
$$

where $f_{k m i n}$ is the minimum, and $f_{k \max }$ is the maximum value of objective $f_{k}$.

The best compromise solution is then determined by the decision maker. A conservative deci- 
sion maker tries to minimise the maximum dissatisfaction for all objectives [35], i.e., to maximise the satisfaction of both the DSO and the EV aggregator. Hence, the final best compromise solution can be found as:

$$
\max _{N_{S}}\left(\min _{k=1}^{2}\left(\mu_{k}\left(X_{n}\right)\right)\right.
$$

By using this criteria, it could be interesting for the decision maker to arrange the Pareto solutions in a descending order, and obtain a priority list of possible schedules. In case the DSO is not interested in the best possible solution, but only requires EV schedules for which the losses are below a certain threshold, the robust multi-objective problem (27) is solved for a fixed value of $\epsilon$.

\section{Test Case}

\subsection{Distribution grid}

The proposed methodology is tested on a real semi-urban low-voltage distribution grid located in Zealand, Denmark, which is modelled based on the information and measurement data provided by a Danish DSO [13]. The described optimisation model is applied to a feeder which is radially run and connected to the $10 \mathrm{kV}$ MV network through a typical $400 \mathrm{kVA}$ distribution transformer with the assumption that the voltages of the MV slack bus are kept at 1 p.u., so $\pm 10 \% U_{n}$ is completely available for $\mathrm{LV}$ regulation $\left(V_{i, t, \min }^{\phi}=0.9 U_{n}, V_{i, t, \max }^{\phi}=1.1 U_{n}\right)$. As seen in Fig. 1 , the observed feeder has 43 residential houses which are three-phase connected with the nominal phaseto-neutral voltage $U_{n}=230 \mathrm{~V}$. The feeder is composed of 13 line segments between $25 \mathrm{~m}$ and 112 $\mathrm{m}$ in length, all of the same cable type with $X / R=0.37$. This corresponds to typical LV grid parameters, e.g., similar to the ones of the CIGRE European LV benchmark network [36]. There are three additional feeders under the same transformer station which have been modelled as an aggregated load due to the lack of data for individual households. All houses in the observed feeder are equipped with smart meters, so individual consumption profiles are based on real metering data with an hourly sampling rate. Consumption values are based on three-phase measurements with no insight into individual phase fractions, so, based on the DSO's experience, it is assumed that the phase unbalance is distributed in 40\%:30\%:30\% ratio. Additionally, the measured data does 

response flexibility parameter $\xi_{i}$ is assumed to be $10 \%$ for all nodes.

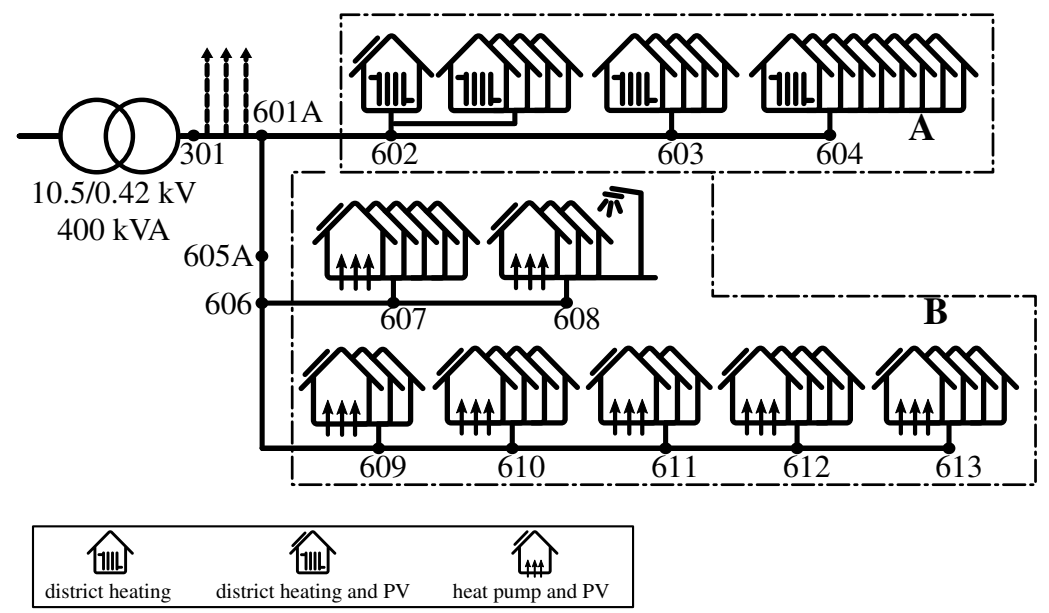

Fig. 1: Schematic overview of the network topology. data.

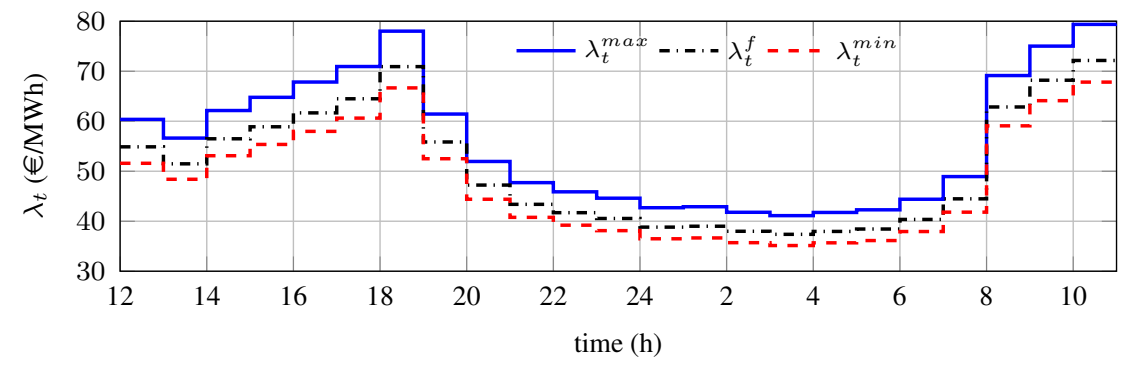

Fig. 2: Forecasted electricity price and uncertainty bounds for the observed 24 hour period.

A characteristic 24-h period has been chosen based on the available historic data to represent the extreme case: a winter day with high residential consumption and almost no PV production. One should note that the chosen period is from 15/01/2013 12:00 until 16/01/2013 11:00 in order to include the night time when EVs are generally available. However, other time windows can be chosen as well. The forecasted electricity price and respective uncertainty range are shown in Fig. 2. These values can be found using time series models such as ARIMA [31] based on historic 


\subsection{Electric vehicle}

It is assumed that Mode 2 charging infrastructure [37] is used for EV charging with a single-phase 16 A connection plug, i.e., $3.7 \mathrm{~kW}$ under nominal voltage conditions. For the sake of simplicity, all EVs are assumed to be a Nissan Leaf with a $24 \mathrm{kWh}$ battery and a constant EV charging efficiency of $80 \%$ [38]. Nevertheless, this assumption does not influence the model's generality as various vehicle types can easily be included. $15 \mathrm{EVs}$ in total are randomly distributed across the observed feeder resulting in an overall $35 \%$ penetration rate. There are 2 vehicles on phase $a$ and 4 on phase $b$ in area $\mathrm{A}$, whereas the distribution in area $\mathrm{B}$ is 2 EVs on phase $a, 3$ on phase $b$, and 4 on phase c. The probability of an EV being plugged-in is taken from the data derived in [39], whereas the initial SOC is taken from the Test-en-EV program where 184 vehicles were distributed to 1600 different Danish families over a three year period [40]. Each vehicle is assigned a random initial SOC, plug-in time and plug-out time as follows:

$$
\begin{gathered}
t_{\text {start }, i}^{\phi E V} \sim \operatorname{GEV}(17.3,0.85,-0.06) \\
t_{\text {end,i }}^{\phi E V} \sim \operatorname{Weibull}(7.67,21.83) \\
S O C_{0, i}^{\phi E V} \sim \mathcal{N}\left(49 \%,(4 \%)^{2}\right)
\end{gathered}
$$

Then, $t_{s t a r t, i}^{\phi E V}$ and $t_{e n d, i}^{\phi E V}$ are rounded to the closest integer value as the simulation time step is chosen to be 1 -h due to the available consumption data. One should note how the initial SOC, the arrival and the departure time are input parameters which are estimated by the EV aggregator. Here, the generalised extreme value distribution is used for the EV arrival time, whereas the Weibull distribution is utilised for the departure time. However, the model generality is not influenced by this choice as other probability distributions can be included as well.

Morevoer, EV reactive power limits are assumed to be $\pm 1.23 \mathrm{kVAr}$ which corresponds to $\cos \phi=0.95$ (ind./cap.) [12]. For comparison, commercially available PV inverters from SMA Solar Technology have the possibility to modulate the reactive power up to $\cos \phi=0.8$ (ind./cap.). It is also assumed that EVs can provide reactive power support only if they are charging, so they cannot act as constant capacitor banks whenever plugged-in. To avoid optimization infeasibility 
due to EVs with short connection time and low initial SOC, a pre-screening is conducted. More precisely, if the estimated connection time is lower than the time necessary for dumb charging, it is assumed there is no active power flexibility from the vehicle. Hence, the charging rate is set to the nominal value and the corresponding active power variable is excluded from the decision variables. Yet, EV reactive power remains a decision variable if available.

\subsection{Scenario overview}

Five scenarios are defined in order to analyse EV potential for charging cost minimisation and concurrent grid support.

1. Scenario I: This case is conducted to provide a basis for comparison between various scenarios. It is assumed that there is no flexibility in the grid $\left(\xi_{i}=0,(P / Q)_{i, t}^{\phi D \text { new }}=(P / Q)_{0, t}^{\phi D}\right)$, no optimisation is performed and EVs charge as soon as they are plugged-in until the battery is completely full $\left(P_{0, i, t}^{\phi E V}=P_{i, \max }^{\phi, E V}, Q_{i, t}^{\phi E V}=0\right)$. The constraint to be satisfied are (1) to (17), whereas the decision variables are limited to load flow variables, i.e., $\mathbf{D V} \mathbf{V}_{I}=$ $\left\{V_{i}^{\phi}, \delta_{i}^{\phi},(P / Q)_{i, t}^{\phi G}\right\}$. Since there is no independent decision variable, the objective function can be chosen as (21) or (27).

2. Scenario II: The nominal optimisation problem (21) is considered without any price uncertainty and using the optimal scheduling as follows:

- IIa: Multi-objective optimisation is performed by optimising only EV active power under constraints (1) to (5) and (9) to (15). This implies that $\mathbf{D V} \mathbf{V}_{I I a}=\mathbf{D V}_{I} \cup\left\{P_{i, t}^{\phi E V}\right\}$.

- IIb: Multi-objective optimisation is performed by optimising both EV active and reactive power under constraints (1) to (5) and (9) to (17). This implies that $\mathbf{D} \mathbf{V}_{\text {IIb }}=\mathbf{D} \mathbf{V}_{I I a} \cup$ $\left\{Q_{i, t}^{\phi E V}\right\}$.

- IIc: Multi-objective optimisation is performed by optimising both EV active and reactive power combined with other demand response resources under constraints (1) to (17). This implies that $\mathbf{D V} \mathbf{V}_{I I c}=\mathbf{D} \mathbf{V}_{I I b} \cup\left\{P_{i, t}^{\phi \text { Dnew }}\right\}$.

3. Scenario III: The multi-objective optimisation problem (27) is considered with price uncer- 
tainty and using the optimal scheduling as follows:

- IIIa: The decision variables are the same as in case IIb, i.e., $\mathbf{D} \mathbf{V}_{I I I a}=\mathbf{D V}_{I I b}$ under constraints (1) to (5) and (9) to (17).

- IIIb: The decision variables are the same as in case IIc, i.e., $\mathbf{D} \mathbf{V}_{I I I b}=\mathbf{D V}_{\text {IIc }}$ under constraints (1) to (17).

The simulations are done using GAMS software with the commercial CONOPT solver (which is well suited for models with very nonlinear constraints) on a notebook with a 2.6-GHz Intel(R) Core(TM) i7-5600U CPU and 8 GB of RAM, taking in average 6-20 seconds for solving one optimisation problem depending on the conducted scenario. In large scale networks, it would be beneficial to use Benders decomposition techniques [41] or task parallelism. The stop criteria for the optimisation is given as the CONOPT's default tolerance value of $10^{-7}$. It is worth noting that the formulated problem is highly non-convex and for such the solver converges to a local optimum which is not necessarily the global one. For Scenarios IIIa and IIIb, the value of $\Gamma$ represents the conservativeness degree which is set by the decision maker. Simulations have been done for all values of $\Gamma=0 \rightarrow 24$.

\section{Simulation Results}

1. Scenario I: In case of uncontrolled charging, total $\mathrm{EV}$ cost equals to $13.58 €(\Gamma=0)$ and total daily active losses are $160.66 \mathrm{kWh}$. The values of possible EV cost for different degrees of uncertainty are given in Table 1. It can be noted how the possible cost would increase from $13.58 €$ to $20.96 €$. The total losses remain the same for all $\Gamma$ as EV schedules are not controlled.

2. Scenario II: Pareto fronts obtained for the nominal optimisation problem of Scenarios IIaIIc are given in Fig. 3 with the best compromise solutions emphasised with filled red shape. Foremost, it is obvious that introducing EV reactive power flexibility has beneficial impact on the grid as the Pareto optimal front moves towards the utopia point, which is even more improved in scenario IIc where demand response is added. It is interesting to notice how the 
Table 1: Total EV cost $(€)$ versus conservativeness degree $(\Gamma)$ for the base scenario I and robust scenarios IIIa and IIIb.

\begin{tabular}{cclcc}
\hline \hline \multirow{2}{*}{$\Gamma$} & \multirow{2}{*}{ Scenario I } & \multicolumn{2}{l}{ Scenario III (best compromise) } \\
IIIa & IIIb & $\begin{array}{c}\text { Scenario III }\left(f_{1}=\epsilon_{\max }\right) \\
\text { IIIb }\end{array}$ \\
\hline 0 & 13.58 & 8.97 & 9.07 & 8.83 \\
2 & 14.19 & 9.35 & 9.25 & 9.18 \\
4 & 14.81 & 9.55 & 9.47 & 9.46 \\
6 & 15.42 & 9.75 & 9.68 & 9.67 \\
8 & 16.03 & 9.81 & 9.84 & 9.70 \\
10 & 16.65 & 9.86 & 9.84 & 9.70 \\
12 & 17.26 & 9.87 & 9.92 & 9.70 \\
14 & 17.88 & 9.87 & 9.92 & 9.70 \\
16 & 18.49 & 9.87 & 9.92 & 9.70 \\
18 & 19.11 & 9.87 & 9.92 & 9.70 \\
20 & 19.72 & 9.87 & 9.92 & 9.70 \\
22 & 20.34 & 9.87 & 9.92 & 9.70 \\
24 & 20.96 & 9.87 & 9.92 & 9.70 \\
\hline \hline
\end{tabular}

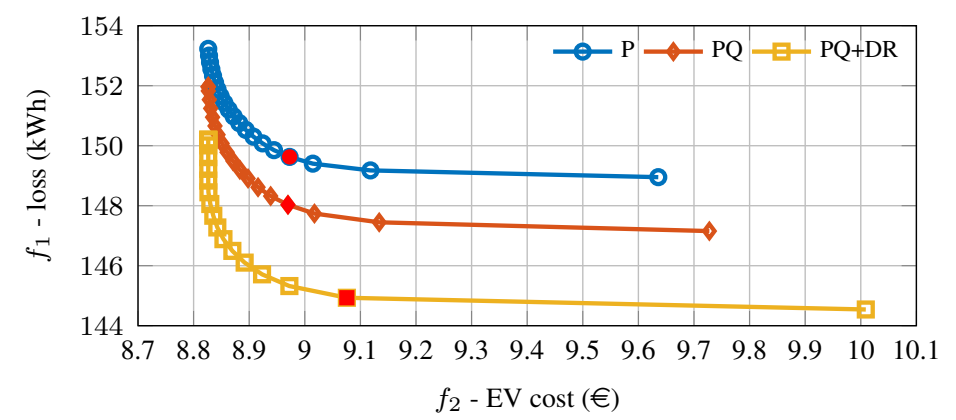

Fig. 3: Obtained Pareto optimal fronts for the nominal optimisation problem in scenarios IIa to IIc $(\Gamma=0)$.

maximum EV cost value is increased by adding more flexibility to the system. One of the reasons is EV reactive power dependency on active power. As EV reactive power influences the losses, but it is only available when EVs are charging, the minimum losses are obtained if part of the charging is shifted to more expensive hours when there is a greater need for reactive power support. The objective functions' values for the best compromise solutions of each scenario and the relative values compared to the uncontrolled charging Scenario I are given in Table 2. It can be seen that modulating EV active and reactive power benefits both the DSO and the EV aggregator compared to the uncontrolled case. The influence of EV reactive power flexibility does not have a significant impact on the EV charging cost, whereas it has positive influence on the losses when comparing the best compromise solutions. Therefore, if EV reactive power capability would be obligatory and implemented in grid codes 
Table 2: Objective functions' values for Scenario I and the best compromise solutions of scenarios IIa to IIc $(\Gamma=0)$.

\begin{tabular}{ccccccc}
\hline \hline Scenario & $\begin{array}{c}\text { EV } \\
\text { flexibility }\end{array}$ & $\begin{array}{c}\text { Demand } \\
\text { response }\end{array}$ & $\begin{array}{c}\text { EV charging } \\
\text { cost }(€)\end{array}$ & $\begin{array}{c}\text { Losses } \\
(\mathbf{k W h})\end{array}$ & $\begin{array}{c}\Delta \text { EV charging } \\
\text { cost }(\boldsymbol{\%})\end{array}$ & $\begin{array}{c}\Delta \text { loss } \\
(\boldsymbol{\%})\end{array}$ \\
\hline I & - & - & 13.58 & 160.66 & - & - \\
IIa & P & - & 8.96 & 149.63 & -34.0 & -6.9 \\
IIb & PQ & - & 8.97 & 148.03 & -33.9 & -7.9 \\
IIc & PQ & $\pm 10 \%$ & 9.07 & 145.32 & -33.2 & -8.9 \\
\hline \hline
\end{tabular}

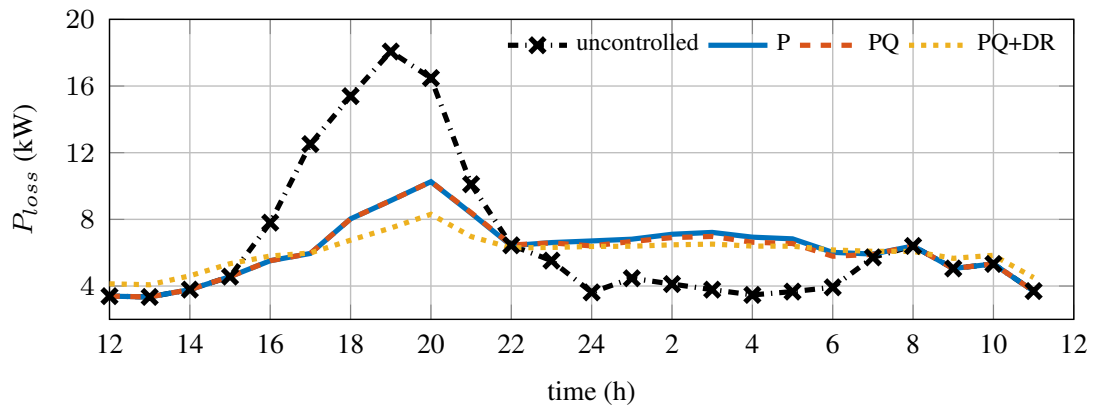

Fig. 4: Active power losses for Scenario I and the best compromise solutions of scenarios IIa to IIc $(\Gamma=0)$.

similarly to the ones for PVs, the DSO would have a greater benefit while the EV aggregator, and consequently EV users, would not be substantially affected. Fig. 4 depicts the active power losses for Scenarios IIa-IIc compared to Scenario I where it is easily noticeable that the highest losses are if EVs remain uncontrolled since EV charging coincides with the peak period. For scenarios IIa-IIc, none of the EVs will charge in the peak period as the electricity price is too high. As depicted in Fig. 6a for several individual EVs, active power schedules depend on the EV connection point. Regardless, all EVs charge during the night resulting in a lower peak load and a reduced need for grid reinforcement. Revisiting Fig. 4, it can be seen that losses in the off-peak period diverge for different scenarios since EVs provide local reactive power support. One should bear in mind that higher EV penetrations would impose a higher total consumption, so introducing the local EV reactive power support could be more beneficial. Moreover, as shown in Fig. 5, even though minimising voltage deviations is not formulated as an objective function, the overall voltages increase with introduction of EV reactive power flexibility since their capacitive behaviour locally supports the grid. Interestingly, even though one would expect only capacitive EV behaviour, inductive behaviour is 


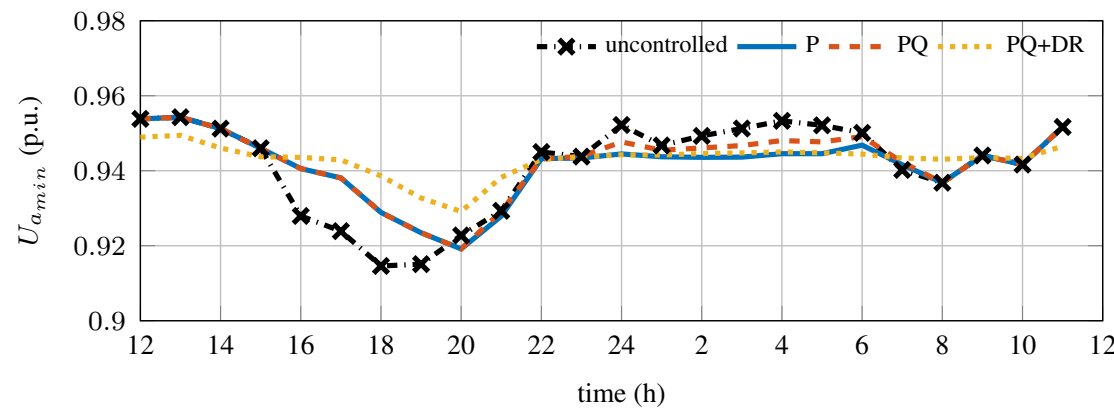

Fig. 5: Minimum phase $a$ voltage values for Scenario I and the best compromise solutions of scenarios IIa to IIc $(\Gamma=0)$.

(a)

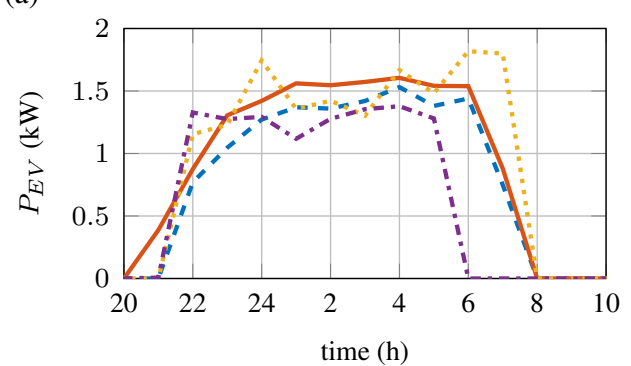

(b)

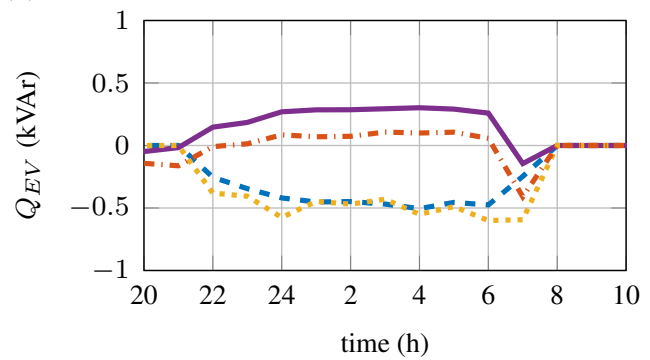

Fig. 6: (a) Active power, and (b) reactive power profiles for selected EVs in scenario IIc.

observed for some vehicles connected in area A as depicted in Fig. 6b. The reason behind are high unbalances in area A due to which several EVs behave inductively to bring the voltages closer together and consequently reduce overall unbalances which influence the losses.

Sensitivity analysis has been conducted for several parameters in Scenario IIc as shown in Fig. 7. First of all, the impact of EV charging efficiency is analysed by changing the value from $80 \%$ to $95 \%$ in $5 \%$ steps. As expected, EV charging efficiency has an impact both on the EV charging cost and the DSO losses. The higher is the efficiency, the greater are the benefits for both entities. Secondly, the impact of maximum EV charging rate has been analysed for three specific rates, i.e. $16 \mathrm{~A}, 32 \mathrm{~A}$ and $63 \mathrm{~A}$, which equal to $3.7 \mathrm{~kW}, 7.4 \mathrm{~kW}$ and $14.5 \mathrm{~kW}$ under the nominal voltage. It can be observed that the EV charging power has an influence only on the maximum losses, whereas the maximum EV cost remains the same since the minimum losses are obtained for a more spread-out EV schedules which are not impacted by the maximum charging rate. However, the higher is the charging rate, the larger are the 
(a)

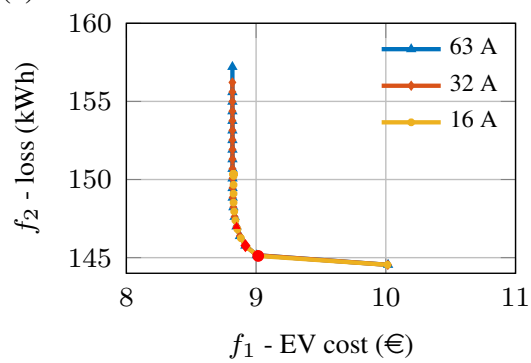

(b)

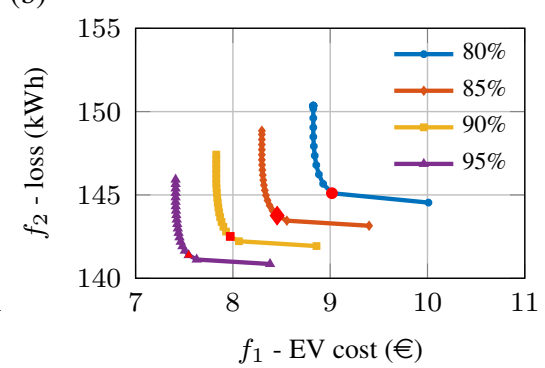

(c)

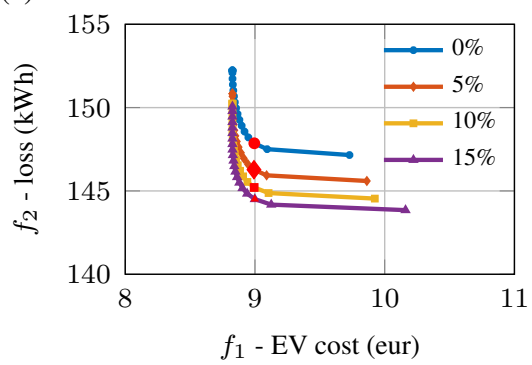

Fig. 7: Impact of (a) maximum EV charging rate, (b) EV charging efficiency, and (c) demand flexibility on Pareto optimal front in Scenario IIc (PQ+DR, $\Gamma=0)$.

3. Scenario III: In Scenario III, the algorithm tries to find a robust solution which minimises the total EV cost for different conservativeness degree $\Gamma$. The numerical values of possible EV costs for the best-compromise solution under different degrees of uncertainty are given in Table 1, whereas Fig. 8 depicts the impact of uncertainty level $\Gamma$ on the Pareto front.

From Table 1, it is clear that the total EV cost is reduced when optimal scheduling is introduced compared to the base Scenario I, but as the uncertainty degree increases, possible EV cost increases as well. The more conservative the decision maker is, the further away will the robust solution be from the nominal case. Regardless, even in the worst case scenario with the most conservative degree $(\Gamma=24)$, the total cost is less than half compared to the base Scenario I. Above a certain degree $\Gamma$, the robust solution stays the same since EVs are scheduled only for several hours in the day, so uncertain prices in the remaining hours will not influence the final EV schedule and cost. One should also note how with the increase of the conservativeness degree, the value of EV flexibility, i.e., the difference between the robust case and the base case, also increases. Comparing Scenarios IIIa and IIIb, it is clear 
(a)

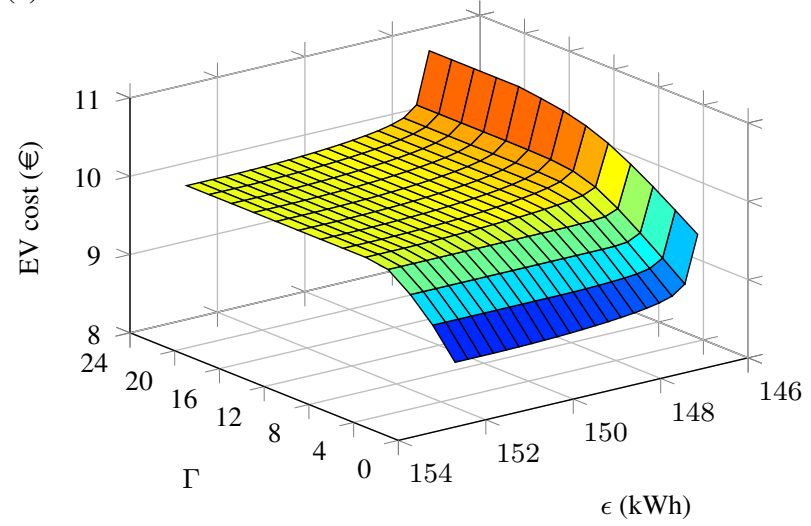

(b)

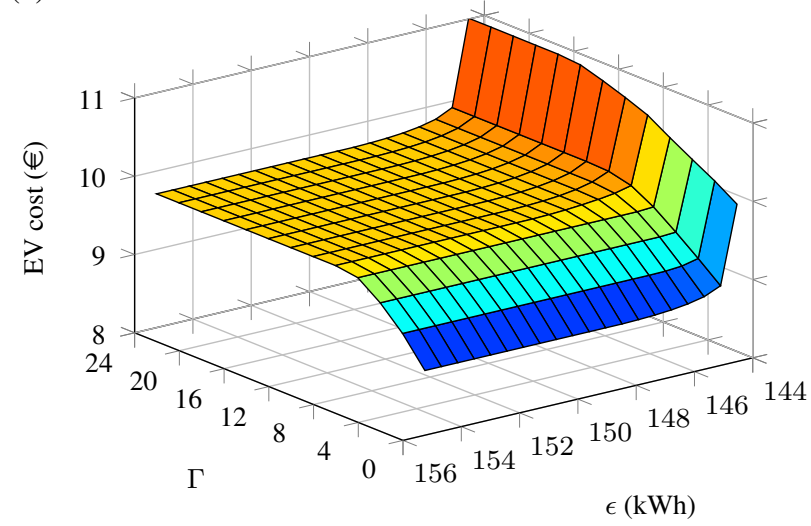

Fig. 8: $\Gamma$ versus $\epsilon\left(f_{1} \leq \epsilon\right)$ versus total EV cost in (a) Scenario IIIa and (b) Scenario IIIb.

(a)

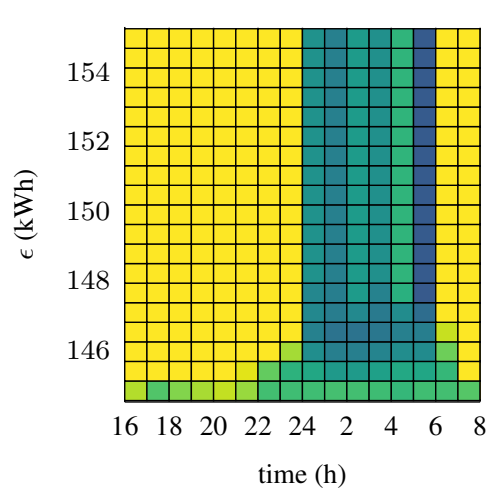

(b)

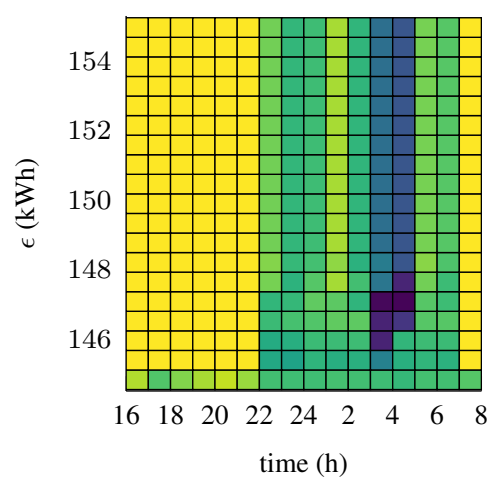

(c)

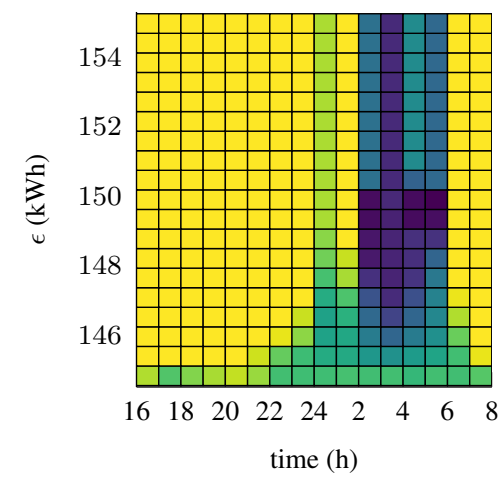

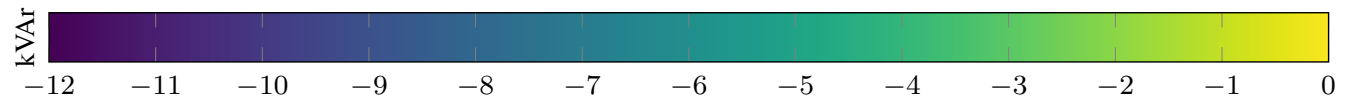

Fig. 9: Total EV reactive power $\sum Q_{i}^{E V}$ versus $\epsilon\left(f_{1} \leq \epsilon\right)$ in Scenario IIIb for (a) $\Gamma=2$, (b) $\Gamma=6$, and (c) $\Gamma=12$.

that minimum losses are lower in Scenario IIIb due to the additional demand response flexibility. Hence, similar to the nominal case, maximum EV cost increases and best-compromise solutions are somewhat higher than in Scenario IIIa, which can also be seen from values in Table 1.

The importance of utilising EV reactive power capability can also be appreciated from Fig. 9 which depicts the cumulative EV reactive power profile in dependence of losses for three values of the conservativeness degree $\Gamma$. It can be observed that reactive power profiles differ since the flexibility is used to satisfy DSO's requirements with respect to losses differently. 
In such way, modulating EV active power can potentially be avoided and, consequently, so can the increase in EV charging cost for various loss values.

In order to check the robustness of the proposed algorithm, a Monte Carlo simulation has been conducted. First, the robust EV schedule is obtained for Scenario IIIb with a set conservativeness degree $\Gamma=12$ and $f_{1}=\epsilon_{\max }$ for which the total $\mathrm{EV}$ cost equals to $9.70 €$ (also seen from Table 1). Next, 10000 samples of price values $\lambda_{t_{1 \rightarrow 24}}$ are generated so that (25b) and (25c) are satisfied. The total EV cost based on the previously obtained decision variables is calculated for each price profile. Fig. 10 clearly shows that all obtained EV cost values are below the value specified by the robust optimisation model marked with a red line $(9.70 €)$. This proves that applying the obtained decision variables ensures the aggregator that the total EV cost will not exceed the obtained robust solution as long as the total price uncertainties remain less than the conservativeness degree $\Gamma$.

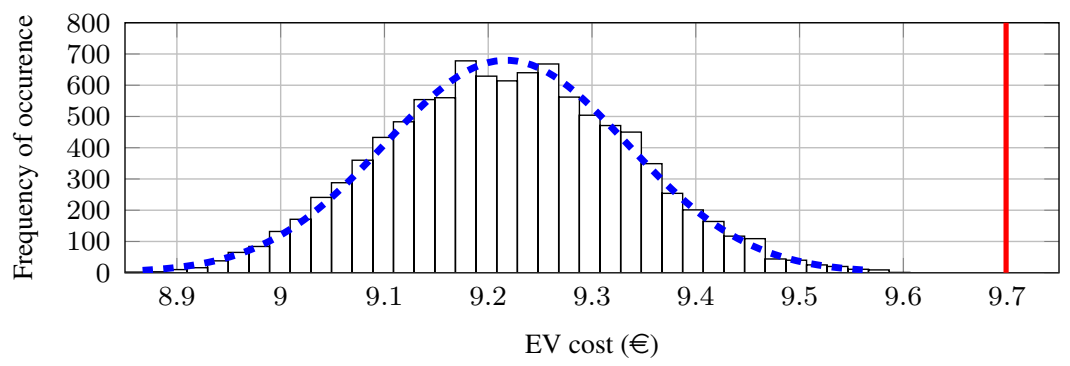

Fig. 10: Results of the Monte Carlo simulation for robustness check in scenario IIIb $(\Gamma=12$, $\left.f_{1}=\epsilon_{\max }\right)$.

\section{Conclusion}

This paper presents a robust multi-objective model for optimal active and reactive EV scheduling in unbalanced distribution networks. Two objective functions have been used in resource scheduling, namely minimisation of losses which represents the DSO's concern, and minimisation of EV charging cost which represents the EV aggregator's main concern. After obtaining a Pareto front, a fuzzy set approach is used to select the best compromise solution, i.e., to minimise the maximum dissatisfaction of both parties. In addition, the impact of EV reactive power capability is investi- 
gated, both on the objective functions' values and on the grid technical constraints.

The method was tested on a real Danish distribution network with a 35\% EV penetration rate. The multi-objective approach was able to obtain the Pareto front with a range of possible solutions, whereas the fuzzy set approach gives a good compromise between the both considered objectives. The robust problem formulation guarantees that the obtained EV cost is optimal as long as the total price uncertainties are lower than the EV aggregator's conservativeness degree. Due to grid unbalances, individual EV schedules differ depending on their connection point and available demand response as well as the set conservativeness degree. It was observed that EV reactive power support can provide benefits for the DSO while not significantly affecting the EV aggregator's cost. By introducing such a capability in grid codes, EVs would be able to provide local grid support resulting in overall improved voltages, decreased losses and less need for reactive power from the external grid.

The question remains what would be the cost of implementing EV reactive power control for voltage support since, without the existence of a voltage market or direct remuneration for voltage regulation, it is difficult to assess the value of such service. The comparison with traditional DSO means, e.g., implementation of capacitor banks, is highly dependent on the analysed grid making it difficult to generalize the economic value, which remains an interesting topic for future work. Additionally, the authors would like to incorporate other sources of uncertainty in the presented model, i.e., demand and PV generation forecast errors and EV user behaviour uncertainty [42], as well as to extend it with EV control in discrete current steps according to contemporary standard IEC 61851 with harmonic analysis. Moreover, future work includes extending the model with application to network planning purposes [43] and to real-time operation [44].

\section{Acknowledgment}

This work is supported by the Danish research projects "NIKOLA - Intelligent Electric Vehicle Integration" under ForskEL kontrakt nr. 2013-1-12088, and "Parker" under ForskEL kontrakt nr. 2016-1-12410. 


\section{References}

[1] P. Siano, "Demand response and smart grids—a survey," Renewable and Sustainable Energy Reviews, vol. 30, pp. 461 - 478, Feb 2014.

[2] T. Shun, L. Kunyu, X. Xiangning, W. Jianfeng, Y. Yang, and Z. Jian, "Charging demand for electric vehicle based on stochastic analysis of trip chain," IET Generation, Transmission Distribution, vol. 10, no. 11, pp. 2689-2698, 2016.

[3] J. Hu, H. Morais, T. Sousa, and M. Lind, "Electric vehicle fleet management in smart grids: A review of services, optimization and control aspects," Renewable and Sustainable Energy Reviews, vol. 56, pp. 1207 - 1226, Apr 2016.

[4] W. Kempton and J. Tomić, "Vehicle-to-grid power implementation: From stabilizing the grid to supporting large-scale renewable energy," Journal of Power Sources, vol. 144, pp. 280294, Jun 2005.

[5] K. Knezović, M. Marinelli, P. Codani, and Y. Perez, "Distribution grid services and flexibility provision by electric vehicles: A review of options," in Power Engineering Conference (UPEC), 2015 50th International Universities, Sept 2015.

[6] M. A. Ortega-Vazquez, F. Bouffard, and V. Silva, "Electric vehicle aggregator/system operator coordination for charging scheduling and services procurement," IEEE Transactions on Power Systems, vol. 28, no. 2, pp. 1806-1815, May 2013.

[7] Y. Tang, J. Zhong, and M. Bollen, "Aggregated optimal charging and vehicle-to-grid control for electric vehicles under large electric vehicle population," IET Generation, Transmission Distribution, vol. 10, no. 8, pp. 2012-2018, 2016.

[8] J. de Hoog, T. Alpcan, M. Brazil, D. A. Thomas, and I. Mareels, "Optimal charging of electric vehicles taking distribution network constraints into account," IEEE Transactions on Power Systems, vol. 30, no. 1, pp. 365-375, Jan 2015. 
[9] J. Franco, M. Rider, and R. Romero, "A mixed-integer linear programming model for the electric vehicle charging coordination problem in unbalanced electrical distribution systems," IEEE Transactions on Smart Grid, vol. 6, no. 5, pp. 2200-2210, Sept 2015.

[10] G. Carpinelli, F. Mottola, and D. Proto, "Optimal scheduling of a microgrid with demand response resources," IET Generation, Transmission Distribution, vol. 8, no. 12, pp. 18911899, 2014.

[11] A. Zecchino, J. Hu, M. Coppo, and M. Marinelli, "Experimental testing and model validation of a decoupled-phase on-load tap-changer transformer in an active network," IET Generation, Transmission Distribution, vol. 10, no. 15, pp. 3834-3843, 2016.

[12] N. Leemput, F. Geth, J. V. Roy, J. Büscher, and J. Driesen, "Reactive power support in residential LV distribution grids through electric vehicle charging," Sustainable Energy, Grids and Networks, vol. 3, pp. 24 - 35, Sept 2015.

[13] K. Knezović and M. Marinelli, "Phase-wise enhanced voltage support from electric vehicles in a danish low-voltage distribution grid," Electric Power Systems Research, vol. 140, pp. 274 $-283,2016$.

[14] H. Liang, B. J. Choi, W. Zhuang, and X. Shen, "Stability Enhancement of Decentralized Inverter Control Through Wireless Communications in Microgrids," IEEE Transactions on Smart Grid, vol. 4, no. 1, pp. 321-331, March 2013.

[15] Smart Energy Demand Coalition, “Mapping Demand Response in Europe Today,” 2015.

[16] M. N. Mojdehi, M. Fardad, and P. Ghosh, "Technical and economical evaluation of reactive power service from aggregated EVs," Electric Power Systems Research, vol. 133, pp. 132 141, Apr 2016.

[17] K. M. Tan, V. K. Ramachandaramurthy, and J. Y. Yong, "Optimal vehicle to grid planning and scheduling using double layer multi-objective algorithm," Energy, vol. 112, pp. 1060 1073, 2016. 
[18] M. Manbachi, A. Sadu, H. Farhangi, A. Monti, A. Palizban, F. Ponci, and S. Arzanpour, "Impact of EV penetration on volt-var optimization of distribution networks using real-time co-simulation monitoring platform," Applied Energy, vol. 169, pp. 28 - 39, 2016.

[19] X. Bai and W. Qiao, "Robust optimization for bidirectional dispatch coordination of largescale V2G," IEEE Transactions on Smart Grid, vol. 6, no. 4, pp. 1944-1954, July 2015.

[20] H. Gao, J. Liu, and L. Wang, "Robust coordinated optimization of active and reactive power in active distribution systems," IEEE Transactions on Smart Grid, vol. PP, no. 99, pp. 1-1, 2017.

[21] B. Jiang and Y. Fei, "Decentralized scheduling of pev on-street parking and charging for smart grid reactive power compensation," in 2013 IEEE PES Innovative Smart Grid Technologies Conference (ISGT), Feb 2013, pp. 1-6.

[22] T. Sousa, H. Morais, Z. Vale, and R. Castro, "A multi-objective optimization of the active and reactive resource scheduling at a distribution level in a smart grid context," Energy, vol. 85, pp. $236-250$, Jun 2015.

[23] J. García-Villalobos, I. Zamora, K. Knezović, and M. Marinelli, "Multi-objective optimization control of plug-in electric vehicles in low voltage distribution networks," Applied Energy, vol. 180, pp. 155 - 168, 2016.

[24] B. Ansari, M. G. Simoes, A. Soroudi, and A. Keane, "Restoration strategy in a self-healing distribution network with dg and flexible loads," in 2016 IEEE 16th International Conference on Environment and Electrical Engineering (EEEIC), June 2016, pp. 1-5.

[25] M. M. A. Abdelaziz, H. E. Farag, E. F. El-Saadany, and Y. A. R. I. Mohamed, "A novel and generalized three-phase power flow algorithm for islanded microgrids using a newton trust region method," IEEE Transactions on Power Systems, vol. 28, no. 1, pp. 190-201, Feb 2013.

[26] E. Sortomme, A. I. Negash, S. S. Venkata, and D. S. Kirschen, "Voltage dependent load models of charging electric vehicles," 2013 IEEE Power Energy Society General Meeting, pp. 1-5, Jul 2013. 
[27] K. Knezovic, S. Martinenas, P. B. Andersen, A. Zecchino, and M. Marinelli, "Enhancing the role of electric vehicles in the power grid: Field validation of multiple ancillary services," IEEE Transactions on Transportation Electrification, vol. 3, no. 1, pp. 201-209, Apr 2017.

[28] A. Soroudi, M. Ehsan, R. Caire, and N. Hadjsaid, "Hybrid immune-genetic algorithm method for benefit maximisation of distribution network operators and distributed generation owners in a deregulated environment," IET Generation, Transmission Distribution, vol. 5, no. 9, pp. 961-972, September 2011.

[29] K. Deb, Multi-objective optimization using evolutionary algorithms. John Wiley \& Sons, 2001, vol. 16 .

[30] A. Soroudi and T. Amraee, "Decision making under uncertainty in energy systems: state of the art," Renewable and Sustainable Energy Reviews, vol. 28, pp. 376-384, 2013.

[31] A. J. Conejo, J. Contreras, R. Espínola, and M. A. Plazas, "Forecasting electricity prices for a day-ahead pool-based electric energy market," International Journal of Forecasting, vol. 21, no. 3, pp. $435-462,2005$.

[32] D. Bertsimas and M. Sim, “The price of robustness," Operations research, vol. 52, no. 1, pp. 35-53, 2004.

[33] A. Soroudi, P. Siano, and A. Keane, "Optimal DR and ESS scheduling for distribution losses payments minimization under electricity price uncertainty," IEEE Transactions on Smart Grid, vol. 7, no. 1, pp. 261-272, Jan 2016.

[34] K. Paridari, A. Parisio, H. Sandberg, and K. H. Johansson, "Robust Scheduling of Smart Appliances in Active Apartments With User Behavior Uncertainty," IEEE Transactions on Automation Science and Engineering, vol. 13, no. 1, pp. 247-259, Jan 2016.

[35] M. Basu, "An interactive fuzzy satisfying method based on evolutionary programming technique for multiobjective short-term hydrothermal scheduling," Electric Power Systems Research, vol. 69, no. 2, pp. 277-285, 2004. 
[36] K. Strunz, N. Hatziargyriou, and C. Andrieu, "Benchmark systems for network integration of renewable and distributed energy resources," Cigre Task Force C, vol. 6, pp. 04-02, 2009.

[37] IEC 61851-1:2010, "Electric vehicle conductive charging system - Part 1: General requirements," 2010.

[38] A. Kieldsen, A. Thingvad, S. Martinenas, and T. M. Sørensen, "Efficiency test method for electric vehicle chargers," in International Battery, Hybrid and Fuel Cell Electric Vehicle Symposium (EVS29), Jun 2016.

[39] N. H. Tehrani and P. Wang, "Probabilistic estimation of plug-in electric vehicles charging load profile,” Electric Power Systems Research, vol. 124, pp. 133 - 143, 2015.

[40] P. Andersen, "Intelligent electric vehicle integration - domain interfaces and supporting informatics," Ph.D. dissertation, Technical University of Denmark, 2013.

[41] A. M. Geoffrion, “Generalized benders decomposition,” Journal of Optimization Theory and Applications, vol. 10, no. 4, pp. 237-260, 1972.

[42] Y. Cao, T. Wang, O. Kaiwartya, G. Min, N. Ahmad, and A. H. Abdullah, "An EV Charging Management System Concerning Drivers' Trip Duration and Mobility Uncertainty,' IEEE Transactions on Systems, Man, and Cybernetics: Systems, vol. PP, no. 99, pp. 1-12, 2016.

[43] P. Maghouli, A. Soroudi, and A. Keane, "Robust computational framework for mid-term techno-economical assessment of energy storage," IET Generation, Transmission Distribution, vol. 10, no. 3, pp. 822-831, 2016.

[44] S. Nojavan, B. Mohammadi-Ivatloo, and K. Zare, "Optimal bidding strategy of electricity retailers using robust optimisation approach considering time-of-use rate demand response programs under market price uncertainties," IET Generation, Transmission Distribution, vol. 9, no. 4, pp. 328-338, 2015. 